\title{
Biomechanical Study on the Treatment of Acromioclavicular Joint Dislocation by Coracoclavicular Ligament Reconstruction With a Novel EndoButton Plate Combined With Suture Anchor
}

\section{Zhe Song}

Honghui Hospital,Xi'an Jiaotong University https://orcid.org/0000-0001-9156-0094

\section{Chen Wang}

Honghui Hospital,Xi'an Jiaotong University

$\mathrm{Na}$ Yang

Honghui Hospital,Xi'an Jiaotong University

\section{Yangjun Zhu}

Honghui Hospital,Xi'an Jiaotong University

Kun Zhang

Honghui Hospital,Xi'an Jiaotong University

Yusheng Qiu ( $\nabla$ yvshengqiu1957@163.com )

The First Affiliated Hospital,College of Medicine,Xi'an Jiaotong University Anhui Provincial Hospital

\section{Research Article}

Keywords: Acromioclavicular joint, Dislocation, Internal fixation, Biomechanics

Posted Date: June 3rd, 2021

DOI: https://doi.org/10.21203/rs.3.rs-570889/v1

License: (c) (i) This work is licensed under a Creative Commons Attribution 4.0 International License.

Read Full License 


\section{Abstract}

Purpose This study aimed to assess the biomechanical stability of a novel internal fixation system of EndoButton plate combined with suture anchor in treating acromioclavicular joint dislocation in the cadaveric specimens. In addition, it provides a new method for the clinical treatment of acromioclavicular joint dislocation.

Methods Twelve complete shoulder joint specimens were randomly divided into groups A, B, C, and D $(n=3)$. Firstly, a quasi-static non-destructive circulation experiment was carried out of coracoclavicular ligament until its function failed. Four different internal fixation materials were used to reduce and fix the acromioclavicular joint. Group A was treated with $3.5 \mathrm{~mm}$ clavicular hook locking compression plates, Group B with $5 \mathrm{~mm}$ suture anchor Group C with $10 \mathrm{~mm}$ Endo-button plate, and Group D with a novel combination of $5 \mathrm{~mm}$ suture anchor and $10 \mathrm{~mm}$ Endo-button plate. Fluoroscopy was performed to undertake the X-ray of the restored acromioclavicular joint, to evaluate the internal fixation position and acromioclavicular joint reduction. Finally, the shoulder joint was fixed firmly on an electronic universal testing machine $(100 \mathrm{KN})$ with a self-made stationary fixture, to conduct a destructive static tensile mechanical test of each specimen vertically at a $100 \mathrm{~mm} / \mathrm{min}$ load speed. The stress-deformation curve was recorded using a computer connected with the universal mechanical testing machine, and the failure strength and reasons for internal fixation were also recorded.

Results The average load-to-failure of the coracoclavicular ligament in groups A, B, C, and D was 373.4 $\pm 0.57 \mathrm{~N}, 373.6 \pm 0.62 \mathrm{~N}, 374.4 \pm 0.68 \mathrm{~N}$, and $373.9 \pm 0.15 \mathrm{~N}$, respectively ( $P>0.05)$. After internal fixation failure, Group A showed two specimens with clavicular fracture, and one with acromial fracture, with an average load-to-failure of $409.8 \pm 2.92 \mathrm{~N}$. Group B and D showed three specimens with prolapse of anchor, with average load-to-failure of $293.5 \pm 4.10 \mathrm{~N}$ and $374.2 \pm 0.40 \mathrm{~N}$, respectively. Group C showed three specimens with basilar coracoid fracture, with average load-to-failure of $373.2 \pm 2.35 \mathrm{~N}$. Statistical differences existed in the biomechanical load of internal fixation failure among the four groups. Group $D$ was statistically different from Group A and Group B, but not Group C.

Conclusion The newly designed EndoButton plate combined with suture anchor for coracoclavicular ligament reconstruction was found to boast simple operation and has high feasibility. Thus it was found effective in the reduction of acromioclavicular joint and treatment of acromioclavicular joint dislocation and fitted the biomechanical characteristics of the acromioclavicular joint.

\section{Background}

Acromioclavicular joint dislocation is a common clinical joint dislocation that accounts for $2-16 \%$ of total joint dislocations and $12 \%$ of shoulder injuries, mostly occurring in young adults $[1,2]$. Its treatment has been controversial for centuries[3,4].A profound understanding of the anatomy and biomechanics of the acromioclavicular joint enables its current treatment to focus on anatomical reconstruction, to reduce the pain in the shoulder joint, and improve its strength and endurance $[5,6]$. The coracoclavicular ligament is 
a thick and strong stabilizer of the lateral clavicle in the vertical direction $[7,8]$. Therefore, anatomical reconstruction in patients with acromioclavicular joint dislocation should be the reconstruction of coracoclavicular ligament $[9,10]$. Although acromioclavicular joint dislocation is exposed to advanced medical concepts and techniques with much more effective treatment, no method or internal fixation is effective. In this study, a new internal fixation system of suture anchor and EndoButton ( $5 \mathrm{~mm}$ suture anchor with $10 \mathrm{~mm}$ EndoButton plate) was designed according to the anatomical and biomechanical characteristics of the acromioclavicular joint. The biomechanical stability of the cadaveric specimens was tested to provide a new method for clinical treatment of acromioclavicular joint dislocation. A comparative study was also carried out with clinically common internal fixation methods to determine its efficacy in treating acromioclavicular joint dislocation.

\section{Materials And Method}

\section{Experimental materials and equipment}

Experimental materials and equipment included a stationary fixture for shoulder joint specimens and tensile-connection set (self-made); specimen dissecting set; microcomputer control electronic universal testing machine (100KN, Shanghai Hualong Test Instruments Co., Ltd. P.R.China); digital image correlation measurement technology; $3.5 \mathrm{~mm}$ clavicle hook locking compression plate (Naton Medical Group, P.R.China), $5 \mathrm{~mm}$ suture anchor (CONMED Corporation,U.S.A), and $10 \mathrm{~mm}$ EndoButton plate(Arthrex Co., Ltd., U.S.A) (Figure 1).

\section{Specimen materials and production}

Twelve complete shoulder joint specimens were fixed with $5 \%$ formalin solution. These cadaveric specimens were provided by the Human Anatomy Experimental Center of Xi'an Medical College, without information on gender and age as it was disassembled into individual shoulder joints. Redundant soft tissues (deltoid and trapezius) were stripped off from the specimens, the acromioclavicular joint was kept about $8 \mathrm{~cm}$ long near the clavicle, the scapula was kept intact, and the proximal humerus was kept about $20 \mathrm{~cm}$ long. Soft tissues around the ligaments were stripped off to expose the ligaments (oblique and conical ligament), keeping them intact to dissect the acromioclavicular and coracoclavicular ligaments (oblique and conical ligament) (Figure 2).

\section{Experimental method}

Twelve complete shoulder joint specimens were divided randomly into four groups A, B, C, and D ( $n=3)$, and the acromioclavicular ligament was cut-off in each group. First, a quasi-static non-destructive experiment was circulated of coracoclavicular ligament until its function failed. Four different internal fixation materials were used to reduce and fix the acromioclavicular joint. Group A was treated with 3.5 $\mathrm{mm}$ clavicular hook locking compression plates, Group B with $5 \mathrm{~mm}$ suture anchor, and Group C with 10 $\mathrm{mm}$ EndoButton plate. Group D was treated with a novel combination of $5 \mathrm{~mm}$ suture anchor with $10 \mathrm{~mm}$ Endobutton plate. X-ray fluoroscopy of the restored acromioclavicular joint was used to evaluate the 
internal fixation position and acromioclavicular joint reduction (Figure 3). Finally, the shoulder joint was firmly fixed on an electronic universal testing machine $(100 \mathrm{KN})$ with a self-made stationary fixture, to conduct a destructive static tensile mechanical test on each specimen vertically at a $100 \mathrm{~mm} / \mathrm{min}$ load speed. The load-displacement curve was recorded using a computer connected with the universal mechanical testing machine, and the failure strength and reasons for internal fixation were also recorded (Figure 4).

\section{Statistical analysis}

All data were expressed as X $\pm \sigma$ and analyzed using SPSS 19.0 (SPSS, Co., Ltd., U.S.A). Initially, the data were analyzed by the homogeneity test of variance. Variance analysis was carried out for grouped comparison of biomechanical load and pairwise comparison between Group D and the other groups. A Pvalue less than or equal to 0.05 was considered statistically significant.

\section{Results}

Average load-to-failure of the coracoclavicular ligament in groups $A, B, C$, and D were $373.4 \pm 0.57 \mathrm{~N}$, $373.6 \pm 0.62 \mathrm{~N}, 374.4 \pm 0.68 \mathrm{~N}$, and $373.9 \pm 0.15 \mathrm{~N}$, respectively $(P>0.05)$. After internal fixation failure, Group A had two specimens with clavicular fracture and one with acromial fracture, with an average loadto-failure of $409.8 \pm 2.92 \mathrm{~N}$. Group B and D had three specimens with prolapse of anchor, with average load-to-failure of $293.5 \pm 4.10 \mathrm{~N}$ and $374.2 \pm 0.40 \mathrm{~N}$, respectively. Group $\mathrm{C}$ had three specimens with a basilar coracoid fracture with an average load-to-failure of $373.2 \pm 2.35 \mathrm{~N}$. The biomechanical load of internal fixation failure among the groups were statistically different. By pairwise comparison, Group $D$ was significantly different from Group $A$ and $B$ in the failure load of internal fixation $(P<0.05)$, but not Group C $(P>0.05)$ (Tables 1 and 2).

\section{Discussion}

Conservative treatments are recommended for Rockwood type I and II dislocation of the acromioclavicular joint. In contrast, surgical treatment is used for high-grade lesions (Rockwood IV-VI) involving coracoclavicular ligament leading to clavicle dislocation. Normal anatomy of the acromioclavicular joint can correct its severe deformity and reduce discomfort and secondary degenerative changes. Although controversial, surgery is recommended for manual workers with Rockwood type-III dislocation of the acromioclavicular joint [11]. Currently, treatment methods of acromioclavicular dislocation have increased that emphasize the biomechanical principle of acromioclavicular joint reduction. Clavicular hook locking compression plate, suture anchor, and Endobutton plate, with high efficacy in treating acromioclavicular joint dislocation, are commonly used clinical treatment methods. However, they are subject to shortcomings and complications. Thus, a new type of suture anchor combined with EndoButton plate was designed in this study according to the biomechanical characteristics of the acromioclavicular joint to treat acromioclavicular joint dislocation. 
A clavicular hook locking compression plate is clinically the most widely used internal fixation to treat acromioclavicular joint dislocation. Due to several advantages, orthopedic surgeons prefer this method of treatment $[12,13]$. Specifically, it features a complete anatomical design, extra-articular plate placement, no damage to joint, little influence on rotator cuff, firm fixation, and availability in early functional exercise. The present biomechanical study revealed that the tensile strength of Group A (up to $409.8 \mathrm{~N}$ ) was remarkably higher than other groups, as well as the normal coracoclavicular ligament. Although its high tensile strength provides a more stable mechanical environment for the shoulder joint, it changes the normal biomechanics of the acromioclavicular joint; hence in practice, its biomechanical non-compliance. In addition, excessive force on it may lead to a fracture of the clavicle or acromion. It is also accompanied by long-term complications. Some frequently reported complications to include loosening, fracture, and migration of internal fixation, shoulder joint pain, traumatic arthritis, subacromial osteolysis, redislocation following plate removal, Subacromial Impingement Syndrome, and second plate removal. Von Heideken et al. [14] reported shoulder pain in $25.5 \%$ of patients after clavicular hook plate surgery. Therefore, most clavicular hook plates were used in the early stage for temporary internal fixation and removed 6-12 months after the operation, to avoid complications.

In recent years, suture anchor characterized by small trauma, simple operation, strength, biomechanical compliance, small size, low stimulation, high histo-compatibility, and no need for removal have become an alternative to treat acromioclavicular joint dislocation. According to Spencer et al. [15], the coracoclavicular ligament endured $500 \mathrm{~N}$, each suture of the suture anchor at $483 \mathrm{~N}$, and the force required to pull a metal anchor was $1150 \mathrm{~N}$. Therefore, researchers believe that suture anchor provide mechanical support for acromioclavicular joint. However, in this study, the acromioclavicular joint in specimens with a suture anchor was found completely dislocated by tensile force at $200 \mathrm{~N}$. The internal fixation failed at $290 \mathrm{~N}$ as the suture anchor prolapsed from the coracoid process and the suture knot slipped during the experiment. Firstly, this may be because the specimens are old shoulder joints fixed with formalin. Changes in the organic and inorganic matter in the bone and an imbalanced component ratio could lead to the loss in the pull-out strength of the suture anchor and thus the dislocation of the suture anchor. Secondly, the grease in the specimen infiltrates the anchor suture. Therefore, mutual friction of sutures decreases and the suture knot slips. Nevertheless, this study could not prove the efficacy of suture anchor in treating acromioclavicular dislocation, although satisfactory results were reported [16] in treating such dislocation clinically with anchor. Thus more experiments and clinical studies are needed for determining its effectiveness.

Implant materials are increasingly diversified, and thus, implant ligament substitutes are widely used[17]. Among them, the Endobutton plate is popular in the reconstruction of the coracoclavicular ligament. Its anatomical reduction, continuous stability, small trauma, few complications, unnecessary second removal, and high biomechanical compliance have favored its use by clinicians [18, 19].Steinbacher et al. [20] compared the Endobutton plate and clavicular hook plate to treat acromioclavicular joint dislocation. They found that the Endobutton plate made patients much less susceptible to complications with higher satisfaction rates and lower social-economic consumption than clavicular hook locking compression plate. Besides, the Endobutton plate was found to be more in line with the biomechanics of 
coracoclavicular ligament, as well as acromioclavicular joint, because its average tensile strength $(373.2 \mathrm{~N})$ is closer to the biomechanical data of normal coracoclavicular ligament ( $374.6 \mathrm{~N})$. In the tensile test, acromioclavicular joint reduction with the Endobutton plate showed high fatigue resistance and effectiveness. Also, the acromioclavicular joint did not present any subluxation or dislocation but slight movement until a basilar coracoid fracture resulted in the failure of internal fixation. Such a slight movement fitted the characteristics of the acromioclavicular joint. However, the TightRope Endobutton by Arthrex used in this study has a complex operation and requires high accuracy of the bone tunnel. Its repeated correction of bone tunnel significantly raised the incidence of coracoid process fracture, damaging peripheral nerves and blood vessels.

By analyzing these treatment methods, a new internal fixation material -suture anchor combined with Endobutton - was designed according to the advantages of the two materials. (Fig. 2d). This method replacing the Endobutton plate with an anchor at the end of the coracoid process is consistent with the biomechanical properties of the acromioclavicular joint, that reduces the difficulty of operation, avoids the establishment of the bone tunnel, and lowers the incidence of coracoid process fracture, and the risk of peripheral neurovascular injuries. Our study showed that suture anchor-Endobutton could provide an average tensile strength of $374.2 \mathrm{~N}$, not different from Endobutton plate $(p>0.05)$, but closer to the biomechanical data of the normal coracoclavicular ligament $(374.6 \mathrm{~N})$. Its tensile test revealed that the new material shared biomechanical properties with Endobutton. The acromioclavicular joint moved slightly. The clavicle and acromion were kept in a position suitable for anatomy, without dislocation or subluxation, until the anchor was pulled-out from the coracoid process, and the internal fixation failed. Such a slight movement conformed to the characteristics of the acromioclavicular joint, which is much easier to manipulate than Endobutton. However, its tensile properties were insignificantly higher than Endobutton plate because changes in the organic and inorganic matter in the coracoid bone and the imbalanced proportion of components reduced the pull-out strength of the suture anchor. Theoretically, the normal coracoid bone can effectively hold suture anchor and increase the tensile strength of the suture anchor-EndoButton plate. Therefore, this study suggests that the new internal fixation system is effective in treating acromioclavicular joint dislocation.

The newly designed suture anchor-Endobutton plate for coracoclavicular ligament reconstruction with high feasibility that boasts simple operation is effective in reducing acromioclavicular joint and treatment of acromioclavicular joint dislocation and fits the biomechanical characteristics of the acromioclavicular joint.

The specimens in the study were old shoulder joint specimens fixed with $5 \%$ formalin solution, that cannot represent fresh specimens considering prolonged fixation and dehydration of their ligaments and bones, which explains the errors in the measured data.

\section{Abbreviations}

Not applicable. 


\section{Declarations}

\section{Ethics approval and consent to participate}

This study was approved by the Institutional Review Committee of Honghui Hospital, Xi'an Jiaotong University. All procedures involving human participants were conducted in accordance with the ethical standards of the institutional and/or national research committee and with the 1964 Declaration of Helsinki and its later amendments or comparable ethical standards. All patients signed informed consent forms prior to the study.

\section{Consent for publication}

Not applicable.

\section{Availability of data and materials}

We state that data will not be shared because all raw data were used to prepare the figures included in the article.

\section{Competing interests}

The authors declare that they have no competing interests.

\section{Funding}

This research had received Shaanxi Social Development Science and Technology Project (2015SF143).

\section{Authors' contributions}

ZS, KZ and YSQ designed the study. CW and NY collected the data. ZS, CW and NY performed the statistical analysis and interpreted the results. ZS and YSQ drafted the manuscript. The final manuscript was approved by all authors.

\section{Acknowledgments}

The authors wish to thank the medical staff of Human Anatomy Experimental Center of Xi'an Medical College where all of the studies were performed.

\section{References}

1. Tauber M., (2013) Management of acute acromioclavicular joint dislocations: current concepts[J]. Archives of Orthopaedic \& Trauma Surgery, 133(7):p.985-995. DOI:10.1007/s00402-013-1748-z.

2. Cisneros L.N. , Reiriz J.S.,(2017) Management of chronic unstable acromioclavicular joint injuries[J]. Journal of Orthopaedics and Traumatology, 18(4):p.305-318. DOI:10.1007/s10195-017-0452-0. 
3. Modi C S., Beazley J., (2013) Controversies relating to the management of acromioclavicular joint dislocations[J]. Bone and Joint Journal, 95-B(12):p.1595-1602. D0Iه10.1302/0301620X.95B12.31802.

4. Petri M., Warth R.J., (2016) Clinical Results Following Non-operative Management for Grade III Acromioclavicular Joint Injuries: Does Eventual Surgery Affect Overall Outcomes?[J]. Arthroscopy: The Journal of Arthroscopic and Related Surgery, p.740-746. DOI:10.1016/j.arthro.2015.11.024

5. Mazzocca A.D., Arciero R.A., (2007) Evaluation and treatment of acromioclavicular joint injuries.[J]. Am J Sports Med, 35(2):p.316-329. DOI:10.1177/0363546506298022.

6. Simovitch R.. (2009)Acromioclavicular joint injuries: diagnosis and management[J]. J Am Acad Orthop Surg, 17(4):p.207-219. DOI:10.1098/rsbl.2003.0144.

7. Beitzel K , Cote M.P.,(2013) Current concepts in the treatment of acromioclavicular joint dislocations. [J].Arthroscopy the Journal of Arthroscopic \& Related Surgery,29(2): p.387-397.

DOI:10.1016/j.arthro.2012.11.023.

8. Harris R.I., Vu D.H.,(2001) Anatomic variance of the coracoclavicular ligaments[J]. Journal of Shoulder \& Elbow Surgery, 10(6):p.585-588. DOI:10.1067/mse.2001.118480.

9. Horst K., Dienstknecht T., (2013) Operative treatment of acute acromioclavicular joint injuries graded Rockwood III and IV: risks and benefits in tight rope technique vs. k-wire fixation[J]. Patient Safety in Surgery, 7(1):p.18-18. DOI:10.1186/1754-9493-7-18.

10. Walz L., Salzmann G.M., (2008) The anatomic reconstruction of acromioclavicular joint dislocations using 2 Tight Rope devices: a biomechanical study.[J]. American Journal of Sports Medicine, 36(12):p.2398. DOI:10.1177/0363546508322524.

11. Beris A., Lykissas M., (2013) Management of acute acromioclavicular joint dislocation with a doublebutton fixation system.[J]. Injury-international Journal of the Care of the Injured, 44(3): p.288292. DOI:10.1016/j.injury.2013.01.002

12. Vincent K.R., Vincent H.K.,(2018) Evaluation and Management of Grade III Acromioclavicular Joint Separations[J]. Current Sports Medicine Reports, 17(11):358-

359. DOI:10.1249/JSR.0000000000000530.

13. Younis F., Ajwani S., (2017) Operative Versus Non-operative Treatment of Grade III Acromioclavicular Joint Dislocations and the Use of SurgiLig: a Retrospective Review[J]. Ortop Traumatol Rehabil, 19(6):p.523-530. DOI:10.5604/01.3001.0010.8043.

14. Von Heideken J., Bostr M Windhamre H., (2013) Acute surgical treatment of acromioclavicular dislocation type $V$ with a hook plate: superiority to late reconstruction[J]. Journal of Shoulder and Elbow Surgery, 22(1):p.9-17. DOI:10.1016/j.jse.2012.03.003.

15. Spencer, Jr.EE., (2007) Treatment of grade III acromioclavicular joint injuries: a systematic review.[J]. Clinical Orthopaedics \& Related Research, 455(455):p.38. DOI:10.1097/BL0.0b013e318030df83.

16. O'Reilly-Harbidge S.C., Balogh Z.J.,(2013) Three-point suture anchor repair of traumatic sternoclavicular joint dislocation[J]. Anz Journal of Surgery, 83(11):p.883-886.

DOI:10.1111/ans.12403. 
17. Ceccarelli E., R. Bondi,(2008) Treatment of acute grade III acromioclavicular dislocation: a lack of evidence[J]. Journal of Orthopaedics \& Traumatology Official Journal of the Italian Society of Orthopaedics \& Traumatology, 9(2):p.105-108.

18. Venjakob A.J., Salzmann G.M., (2013) Arthroscopically Assisted 2-Bundle Anatomic Reduction of Acute Acromioclavicular Joint Separations 58-Month Findings[J]. The American Journal of Sports Medicine, 41(3). DOI:10.1177/0363546512473438.

19. Salzmann G.M., Walz L., (2010) Arthroscopically Assisted 2-Bundle Anatomical Reduction of Acute Acromioclavicular Joint Separations[J]. 38(6):p.1179-1187. DOI:10.1177/0363546509355645

20. Gilbert, Steinbacher, (2014) Clavicular Hook Plate for Grade-III Acromioclavicular Dislocation[J]. J Orthop Surg (Hong Kong), 22(3):p.329-332. DOI:10.1177/230949901402200312

\section{Tables}

Table 1 Group comparison of the mechanical load of coracoclavicular ligament rupture and internal fixation failure

\begin{tabular}{lcc}
\hline \multicolumn{1}{c}{ Group } & Coracoclavicular ligament rupture (N) & Internal fixation failure(N) \\
\hline Clavicular hook plate (A) & $373.4 \pm 0.57$ & $409.8 \pm 2.92$ \\
Suture anchor (B) & $373.6 \pm 0.62$ & $293.5 \pm 4.10$ \\
Endobutton Plate (C) & $374.4 \pm 0.68$ & $373.2 \pm 2.35$ \\
Suture anchor-EndoButton Plate (D) & $373.9 \pm 0.15$ & $374.2 \pm 0.40$ \\
P value & 0.211 & 0.000 \\
\hline
\end{tabular}

Table 2 Pairwise comparison of mechanical loads of internal fixation failure

\begin{tabular}{|c|c|c|c|}
\hline Clavicula & 1ook plate (A) & suture anchor (B) & Endobutton (C) \\
\hline Suture anchor-EndoButton Plate (D) & 0.000 & 0.000 & 0.973 \\
\hline
\end{tabular}

\section{Figures}




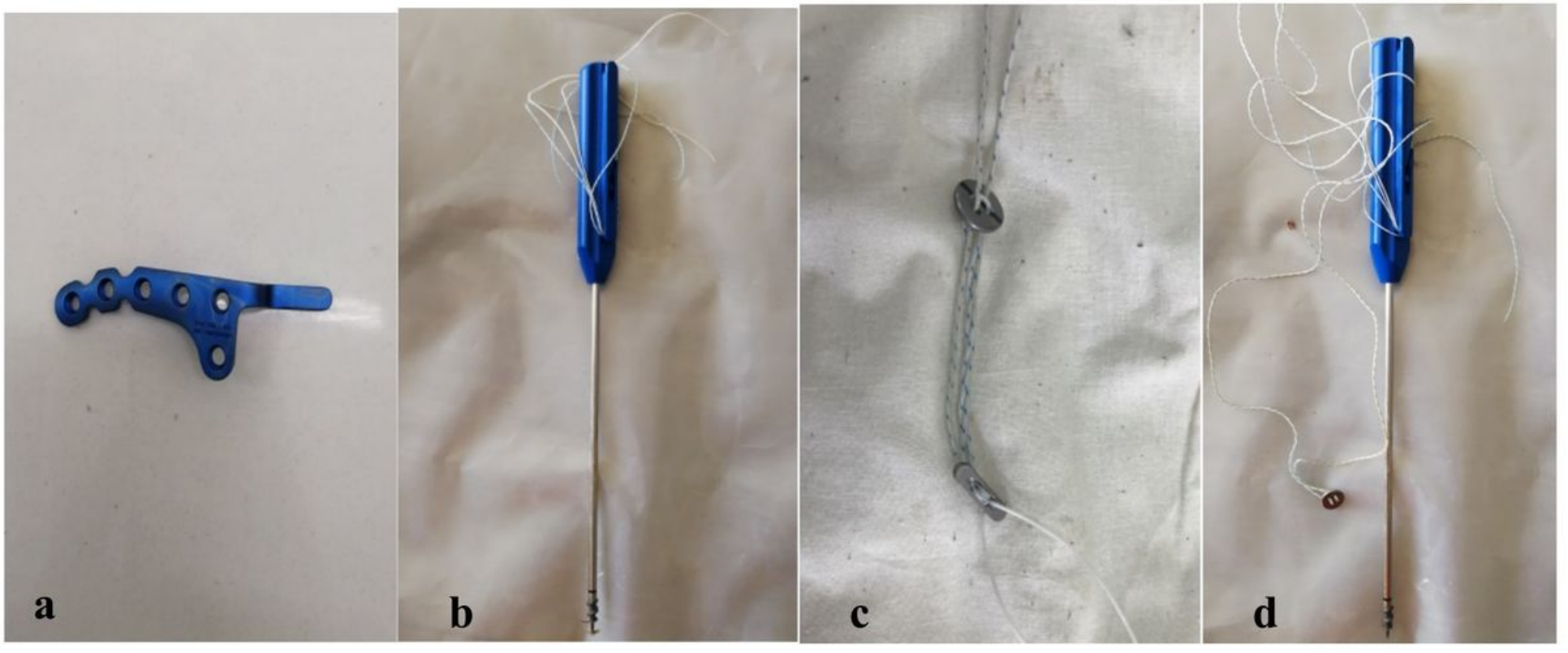

\section{Figure 1}

Internal fixation materials a: Clavicle hook locking compression plate; b: Suture anchor; c: Endobutton plate; d: Suture anchor-EndoButton plate

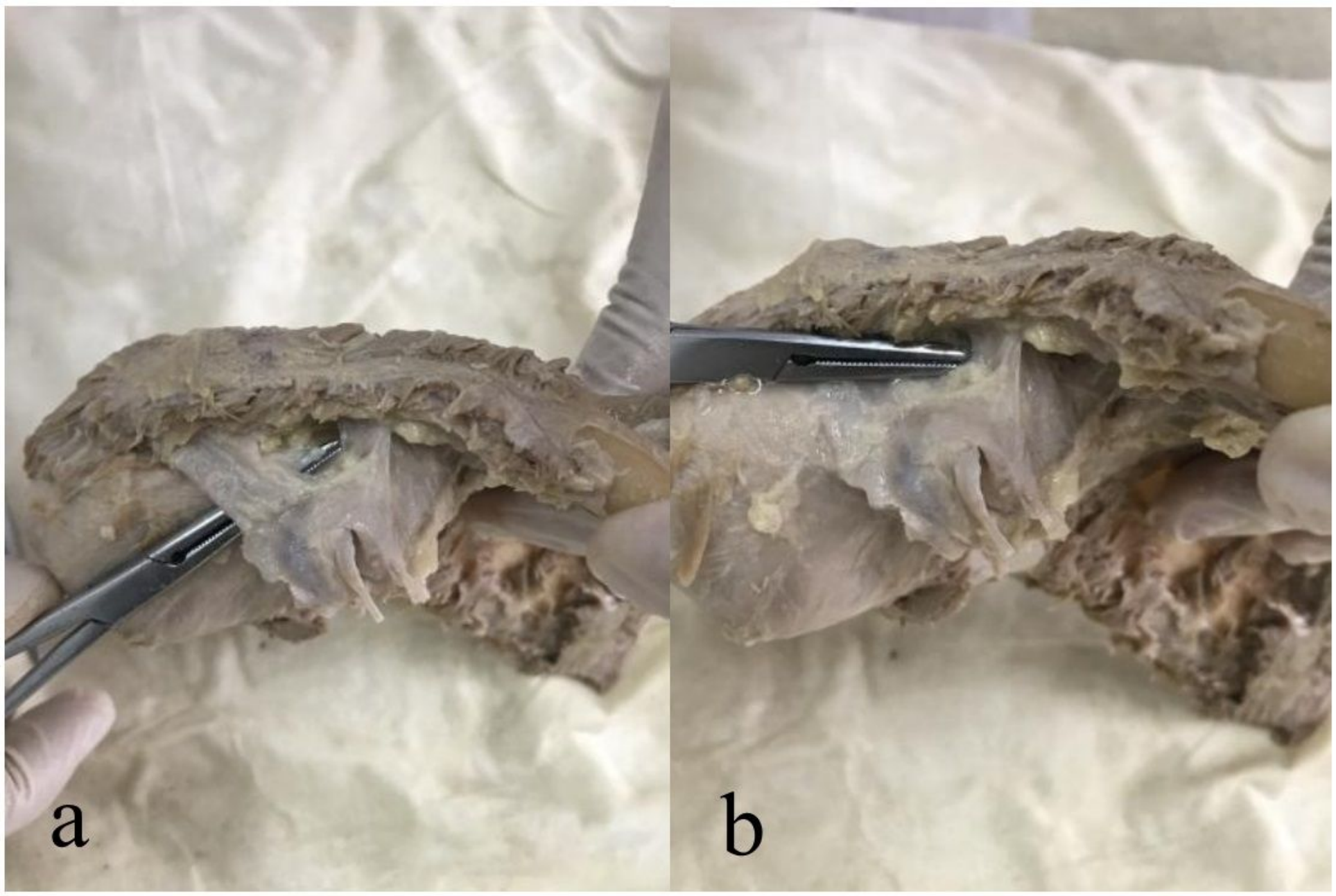

Figure 2 
Cadaveric specimens of coracoclavicular ligament a: Trapezoid ligament; b: Conoid ligament
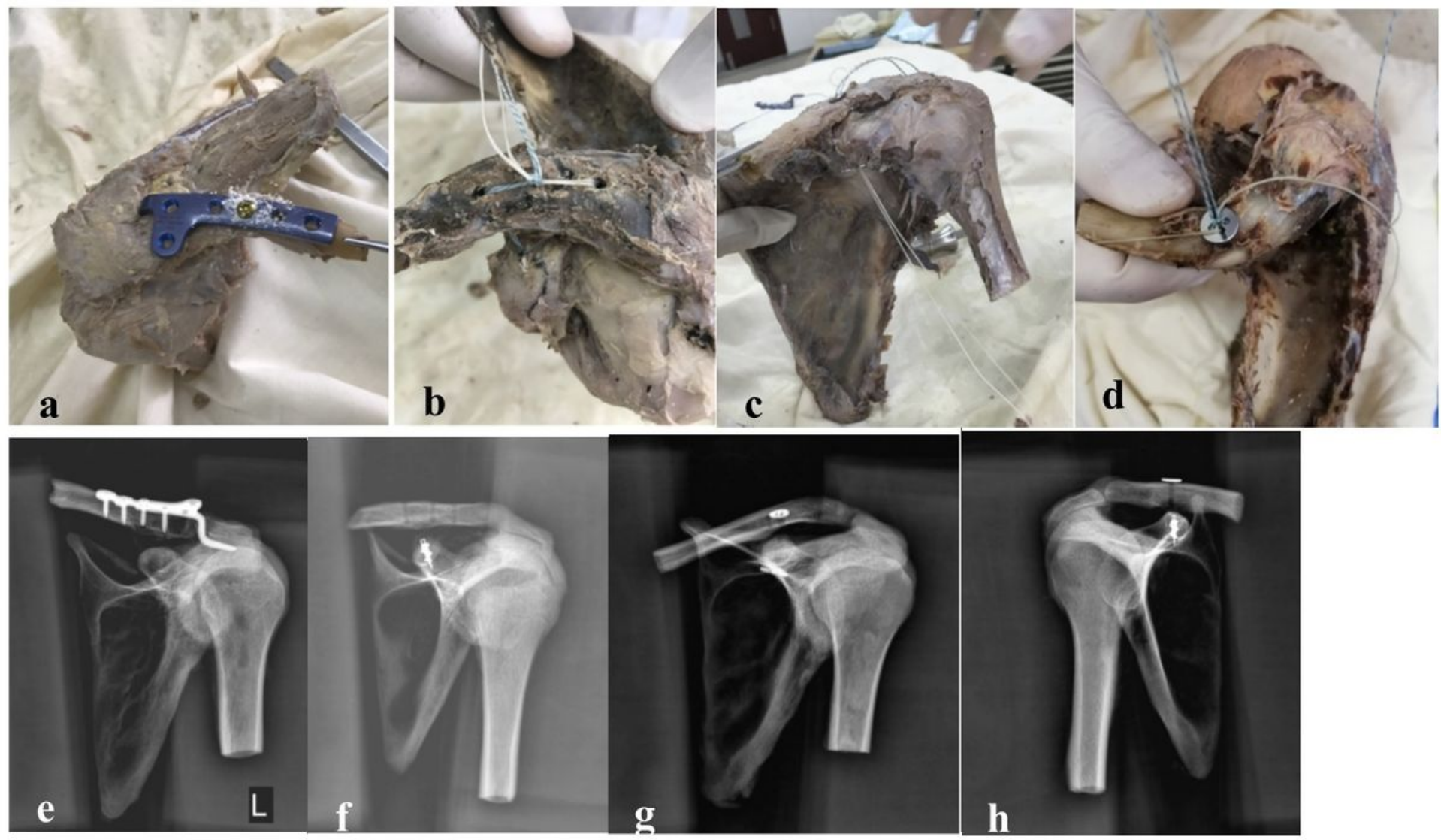

\section{Figure 3}

Reduction, fixation, and X-ray results of acromioclavicular joint a: Clavicle hook locking compression plate; b: Suture anchor; c: Endobutton plate; $d$ : Combination of suture anchor with EndoButton plate; e: Xray results of clavicular hook locking compression plate; $\mathrm{f}$ : X-ray results of suture anchor; $\mathrm{g}$ : X-ray results of EndoButton plate; h: X-ray results of suture anchor-EndoButton plate.
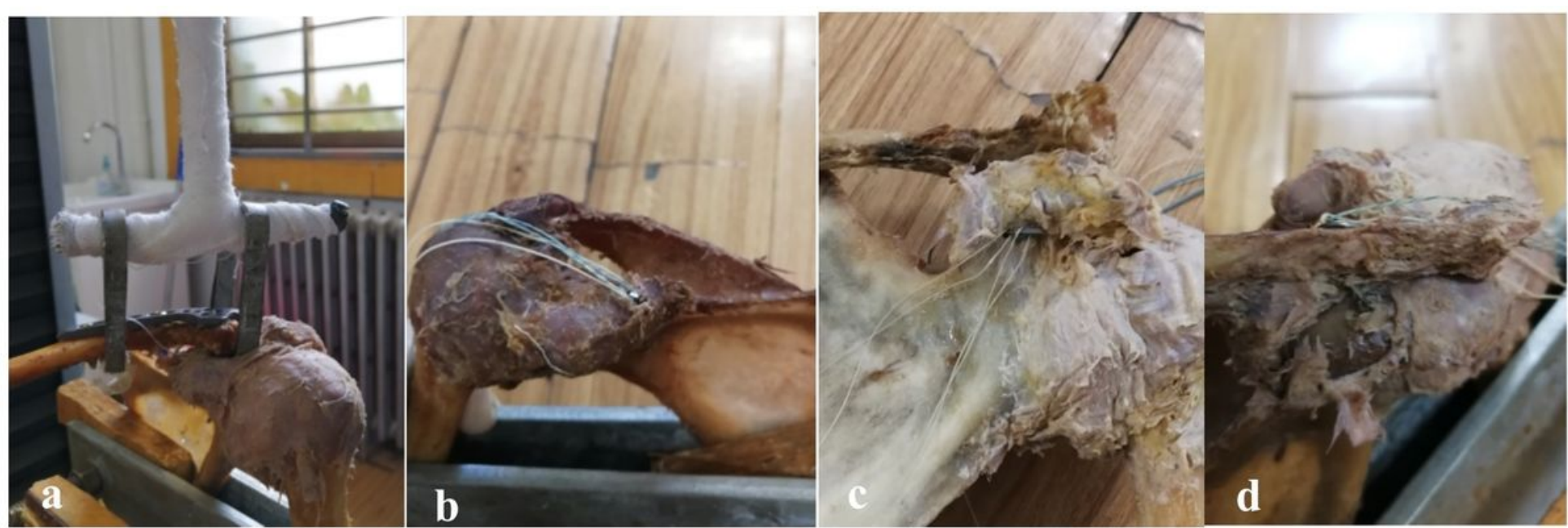

Figure 4 
Causes of fixation failure in each group a. Two specimens failed due to clavicular fracture, and one due to acromion fracture in Group A treated with the clavicle hook locking compression plate; b. In Group B, three specimens failed due to anchor prolapse treated with suture anchor; c. In Group C, three specimens treated with the Endobutton plate failed due to basilar coracoid fracture; $d$. Three specimens failed due to anchor prolapsed in Group D treated with a novel combination of suture anchor with EndoButton plate. 